\title{
Evaluación y Utilidad del Cuestionario DASI (Duke Activity Score Index) para la Estimación de Capacidad Funcional en Población Chilena
}

\author{
Paola Varleta ${ }^{1-2}$, Maríaluisa Von Chrismar ${ }^{1, a}$, Gabriela Manzano ${ }^{1, b}$, Paulina Cisterna ${ }^{2, a}$, Rosario Lopez ${ }^{3, b}$, Ingrid Morales ${ }^{2}$, Denisse Lama $^{1}$, \\ Carlos Navarrete $4, d$. \\ 1. Unidad de Prevención Cardiovascular y Rehabilitación Cardíaca, Centro Cardiovascular, Hospital DIPRECA. \\ 2. Unidad de Cardiología, Servicio de Medicina. Hospital de Carabineros. \\ 3. Unidad de Kinesiología, Hospital Clínico Universidad Católica. \\ 4. Departamento de Matemáticas, Universidad de la Serena. \\ a. Enfermera. \\ b. Kinesióloga. \\ c. Phd.
}

Sin fuente de apoyo financiero externo.

El Duke Activity Status Index (DASI) es un cuestionario inglés utilizado para la estimación de capacidad funcional, que se ha correlacionado con equivalentes metabólicos (METs) y con consumo de oxígeno (VO2max). No existe información de su aplicación en población chilena. El objetivo fue evaluar la utilidad de una versión de DASI traducida al español y adaptada a población local, para predecir capacidad funcional determinada en prueba de esfuerzo máxima.

Método: Se tradujo al español el DASI junto con adaptación de preguntas para población local. El cuestionario fue auto administrado previo a la realización de una prueba de esfuerzo máximo, sólo detenida por síntomas y percepción de esfuerzo de $\geq 17$ /20 en escala de Borg. Se correlacionó METs con VO2max logrados en esfuerzo versus los estimados por DASI.
Resultados: Se incorporaron 480 sujetos (edad x: $50.9 \pm 15.3$ años). La mediana (IQ) de METS estimados por DASI fue 9,2 (8,2-9,8). El valor $\alpha$ de Cronbach y Formula 20 de Kuder Richardson fue 0,72. El cuestionario fue de rápida aplicación. Se observó correlación significativa entre METs (r: 0.44; $\mathrm{p}<0.001$ ) y VO2max (r: 0,$37 ; p<0.001)$ determinados por cuestionario DASI y prueba de esfuerzo máxima.

Conclusión: El DASI traducido y adaptado a población chilena es un instrumento sencillo de completar, que se correlaciona positivamente con capacidad funcional de forma similar a lo descrito en otros países, entregando una herramienta útil para la evaluación y predicción de riesgo cardiovascular.

Palabras Clave: Aptitud Física, Prueba de Esfuerzo, Equivalente Metabólico, Cuestionarios. 


\section{A Spanish version of the DASI questionnaire for estimation of functional capacity and cardiovascular risk applied to a Chilean population}

The Duke Activity Status Index (DASI) is an English questionnaire used to estimate functional capacity and physical fitness that correlates with peak oxygen uptake ( $\mathrm{VO} 2 \max )$ and metabolic equivalents (METs). There is no available information of its application in Chilean population.

Aim: to assess the efficacy of a Spanish version of the DASI questionnaire in predicting functional capacity in a Chilean population.

Methods: DASI was translated into a Spanish version and adapted to local population and culture. The questionnaire was self-administered prior to carrying out a treadmill exercise stress testing, stopped only by symptoms or a perception of stress $\geq 17$ / 20 on the Borg scale. Internal consistency was estimated with two tests. A correlation was performed be- tween the METs and VO2max achieved in treadmill stress testing versus those estimated by DASI.

Results: 480 subjects were enrolled (age $\mathrm{x}: 50.9$ \pm 15.3 years old). The median (IQ) DASI score was 9,2 (8,2-9,8). Both Cronbach's $\alpha$ and Kuder Richardson Formula 20 were 0,72 . DASI was easy and quick to apply. A significant correlation was observed between METS by DASI and those estimated by stress testing ( $r: 0.44: p<0.001$ ); the same was true for the estimation of VO2max (r:0.37: $\mathrm{p}<0.001)$. Conclusion: The Spanish DASI translation adapted to Chilean population is an easy instrument to apply. Results are similar those obtained in other countries in the estimation of functional capacity. Key words: Physical fitness; exercise test; metabolic equivalent; questionnaires. 


\section{Introducción:}

La capacidad funcional (CF) es un concepto que refleja la habilidad de desarrollar actividades de la vida diaria requirentes de metabolismo aeróbico ${ }^{1}$. Una baja $\mathrm{CF}$ se asocia a una baja aptitud cardio-respiratoria, y a mayor riesgo de mortalidad cardiovascular $(\mathrm{CV})^{2}$. La CF se relaciona con el consumo de oxígeno máximo (VO2 max), definido como el producto del gasto cardíaco y la diferencia arterio-venosa de oxígeno ante un ejercicio máximo extenuante ${ }^{1}$. Una manera directa de estimar el VO2 max, es a través de la medición ventilatoria de producción de dióxido de carbono durante el rendimiento máximo en un test cardiopulmonar ${ }^{3}$. Sin embargo, esta determinación del VO2 max no es de fácil acceso en la práctica hospitalaria. Por otra parte, la prueba de esfuerzo (PE) máxima con estimación de los equivalentes metabólicos (METs), es un examen muy solicitado, ${ }^{4}$ como una alternativa indirecta validada de estimación de CF.

La determinación de $\mathrm{CF}$ es útil, pero como no siempre se cuenta con PE, expertos han generado cuestionarios que permiten estimarla en forma indirecta. Dentro de estos cuestionarios destaca el Duke Activity Score Index (DASI), de Hlatky y col., que demostró correlación con VO2max ${ }^{5}$. El DASI consiste en 12 preguntas sobre actividades de la vida diaria, que evalúan cinco ámbitos: cuidado personal, marcha, cuidado de la casa, actividad sexual y actividades deportivas. En Estados Unidos, el DASI es utilizado e incluso ha sido incorporado en un algoritmo de la Asociación Americana del Corazón (AHA), referente a sospecha de enfermedad isquémica en la mujer. 6

En América Latina, existe escasa información sobre DASI. En Brasil, Coutinho-Myrrha y col. lo tradujeron al portugués mostrando correlación con METs en PE máximo $^{7}$. Por otra parte, existe una traducción al español realizada en Colombia que consideró vocablos poco utilizados localmente ${ }^{8}$. En Chile, no disponemos de información sobre la aplicación del DASI. El objetivo del presente estudio fue evaluar la utilidad de una versión de DASI traducida al español adaptada a población chilena, para predecir CF determinada en PE máxima.

\section{Material y metodos:}

\section{Sujetos:}

Estudio de corte transversal con reclutamiento prospectivo de población ambulatoria referida a PE entre abril y diciembre de 2015. Se incluyó todo paciente $\geq 15$ años con capacidad cognitiva y visual para responder cuestio- nario auto administrado y sin antecedente de enfermedad vascular periférica conocida (claudicación intermitente y/o amputación), enfermedad pulmonar crónica, y/o alteración locomotora que limitara ejercicio en cinta móvil. Se excluyó del análisis, todo sujeto que diera respuesta incompleta del cuestionario, y/o que presentara alteraciones al electrocardiograma, arritmias o alza de presión arterial (PA) sistólica $\geq 250 \mathrm{mmHg}$ y/o PA diastólica $\geq 115$ $\mathrm{mmHg}^{9}$, que implicase el término anticipado de la PE. De 601 pacientes enrolados, 121 fueron excluidos del análisis por respuesta incompleta, y 96 sujetos por $\mathrm{PE}$ submáxima, detenida anticipadamente.

El estudio fue aprobado por el comité de ética del Hospital DIPRECA. Todo participante firmó consentimiento informado, previo a la realización del estudio.

\section{Recolección de datos y definición de variables:}

Se efectuó una entrevista por enfermera que incluyó antecedentes demográficos, fármacos en uso, y autor reporte de factores de riesgo cardiovascular (FRCV), hipertensión arterial, dislipidemia, diabetes, y tabaquismo. Se midió peso y talla, y se estimó el índice de masa corporal (IMC). Se definió como hipertenso, dislipidémico o diabético a todo sujeto con diagnóstico médico respectivo, con o sin tratamiento farmacológico. Se consideró tabaquismo, el haber fumado al menos un cigarro en el último mes. Se definió obesidad por un IMC $\geq 30 \mathrm{~kg} / \mathrm{m} 2$.

Los participantes respondieron el DASI por autoadministración previo a la realización de la PE, con el cardiólogo ejecutante ciego al resultado del cuestionario. Se realizó PE en equipo General Electric modelo Marquette CASE System 6.7 con cinta móvil T1200, bajo protocolo Bruce o Naughton, según indicación médica de referencia. Todo estudio se realizó bajo supervisión de cardiólogo. La CF fue determinada a través de METs logrados en PE máxima, limitada por síntomas tales como fatiga, disnea, y/o angina limitante. El esfuerzo máximo durante el ejercicio fue determinado por frecuencia cardíaca $\geq 85 \%$ de la predicha para la edad, y una percepción de esfuerzo máximo de $\geq 17$ en escala de Borg de 20.10

Se utilizaron fórmulas para el cálculo de VO2max a través del tiempo total en minutos alcanzado en la PE. Para el protocolo Naughton, la fórmula utilizada fue VO2 max $(\mathrm{ml} / \mathrm{kg} / \mathrm{min})=1.61 \times \mathrm{T}(\mathrm{min})+3.6$ sin variación por género ${ }^{11,12}$. En el caso del protocolo Bruce se utilizaron fórmulas diferenciadas por género. En hombres VO2 max (ml/ $\mathrm{Kg} / \mathrm{min})=14.8-(1.379 \times \mathrm{T})+\left(0.451 \times \mathrm{T}^{2}\right)-\left(0.012 \times \mathrm{T}^{3}\right)$ $\mathrm{y}$ en mujeres VO2 $\max =4.38 \times \mathrm{T}-3.9$, donde $\mathrm{T}=\mathrm{Tiempo}$ total en la cinta medido como fracción de minuto. ${ }^{13,14}$ 
Tabla 1- Cuestionario DASI versión en Español adaptado a población chilena:

formulación de 12 preguntas con puntaje estimado frente a respuesta positiva

\begin{tabular}{|l|c|}
\hline Actividad & Valor Estimado \\
\hline 1. ¿Puede Usted realizar sus actividades de autocuidado, tales como alimentarse, vestirse, bañarse, o usar el baño? & 2,75 \\
\hline 2. ¿Puede Usted caminar dentro de su casa y alrededor de ésta? & 1,75 \\
\hline 3. ¿Puede Usted caminar una o dos cuadras, sin pendiente? & 2,75 \\
\hline 4. ¿Puede subir un tramo de escalones ( ej: dos pisos de escaleras) sin detenerse? & 5,50 \\
\hline 5. ¿Puede correr una pequeña distancia? & 8,00 \\
\hline 6. ¿Puede realizar trabajos livianos en su casa tales como sacudir el polvo, o lavar platos? & 2,70 \\
\hline 7. ¿Puede realizar trabajo moderado en su casa tal como pasar la aspiradora, barrer los pisos, o acarrear & 3,50 \\
\hline comestibles desde una tienda o mercado? & 8,00 \\
\hline 8. ¿Puede realizar trabajo pesado en su casa tal como el fregado de piso, o levantar o desplazar muebles pesados? & 4,50 \\
\hline 9. ¿Puede hacer trabajos en el jardín tales como rastrillar las hojas, desmalezar o empujar una cortadora de pasto? & 5,25 \\
\hline 10. ¿Puede tener relaciones sexuales? & \\
\hline 11. ¿Puede participar en actividades recreacionales físicas de intensidad moderada como baile entretenido, \\
\hline zumba, jugar tenis (en dobles), jugar golf o lanzar pelotas? & 6,00 \\
\hline 12. ¿Puede participar en deportes intensos como natación, tenis individual, fútbol, baloncesto o esquí? & 7,50 \\
\hline
\end{tabular}

\section{PUNTAJE TOTAL SUMATORIO}

Cálculo VO2max = puntaje total DASI $\times 0.43+9.6 /$ METs = puntaje total DASI $\times 0.43+9.6 / 3.5$

\section{Cuestionario DASI:}

El cuestionario DASI fue traducido al español por los autores con asesoría de un traductor y kinesiólogos. Cada pregunta tenía respuesta dicotómica y un puntaje basado en el costo metabólico asociado. ${ }^{5}$ Sólo si la respuesta era positiva se obtenía puntaje, siendo el puntaje total de 58,2 puntos. Se adaptaron algunas preguntas modificando actividades cotidianas para la población norteamericana por aquellas reconocibles y/o usuales para nuestra cultura. En la Tabla 1 se presenta el cuestionario DASI traducido y adaptado a población local. Con el puntaje DASI se estimó VO2max y METs utilizando fórmulas validadas por Hlatky y $\mathrm{col}^{5}$.

\section{Análisis Estadístico}

Los datos se analizaron con software $\mathrm{R}$ versión 4.0.2. Las variables continuas se expresaron como mediana con rangos intercuartiles (IQR) según la distribución de los datos por prueba Mann-Whitney. Se realizó análisis de la consistencia interna del DASI con prueba alfa de Cronbach y también, con Fórmula 20 de Kuder Richardson, por ser dicotómico. Adicionalmente, se realizó un análisis de carga de FRCV con puntaje DASI y con METs por $\mathrm{PE}$, mediante prueba no-paramétrica de Mann-Whitney.
Se categorizó el número de FRCV como 0-1 vs $\geq 2$, debido al bajo número de pacientes con 3 o más factores. Se efectuó un análisis de regresión lineal simple para estimar correlación entre los valores estimados de VO2max y METs por DASI y por PE máxima. Por observarse variabilidad en el desempeño físico para los puntajes más altos del DASI, se elaboró un análisis de regresión lineal ocupando METs en escala logarítmica, con estimación basada en función de suavizamiento LOESS, homogeneizándose la varianza. Se utilizó un modelo de regresión lineal Bayesiano entre METs y puntaje DASI, para obtener una evaluación de distribución predictiva de METs para valores del DASI, y determinar la probabilidad de obtener un desempeño superior a 3,6 y 9 METs. Se seleccionó el corte de 3, 6, y 9 METS, por corresponder a un nivel leve, moderado y vigoroso de intensidad de actividad física, respectivamente, según el Colegio Americano de Medicina del Deporte ${ }^{15,16}$.

\section{Resultados:}

Se incluyeron 480 pacientes, $37.7 \%$ mujeres. La edad promedio del grupo fue de $51 \pm 5$ años, un $21 \%$ era diabético, $44 \%$ hipertenso, y un $15 \%$ refirió antecedente de enfermedad coronaria conocida. La mayoría de los 
Figura 1.

A.

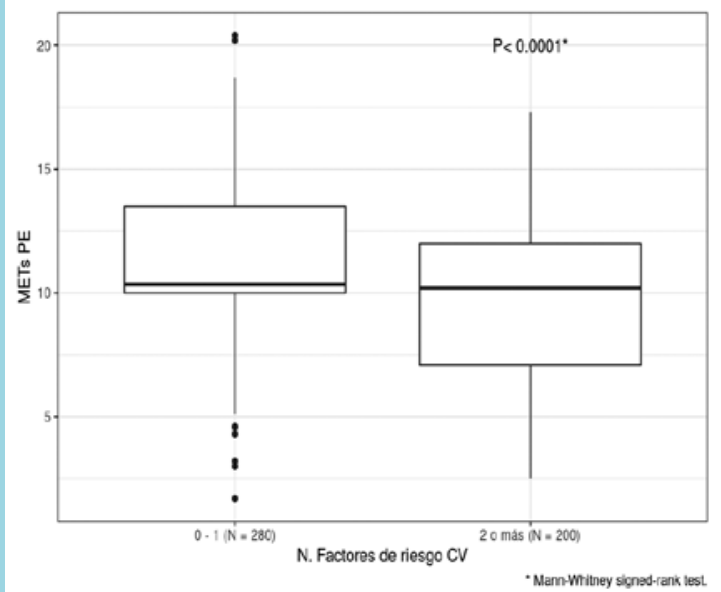

B.

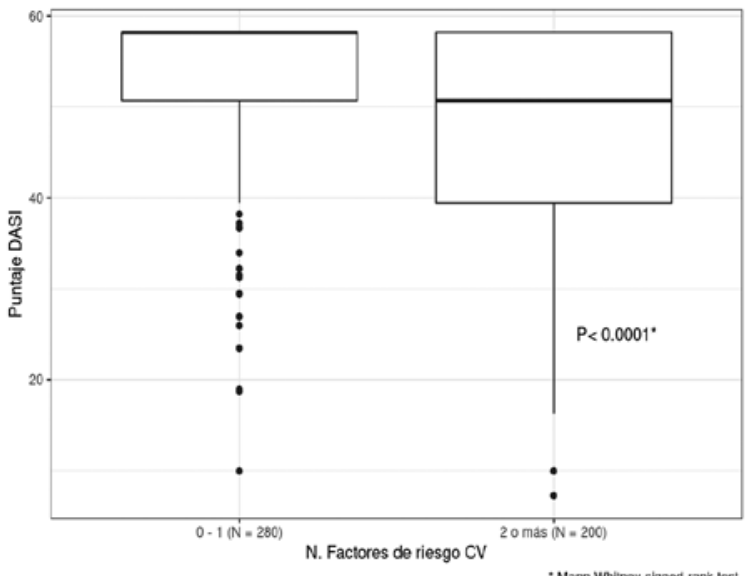

$\cdot$ - Mann-Whiney signed-rank test

Asociación de Carga de Factores de Riesgo Cardiovascular con los Equivalentes Metabólicos (METS) alcanzados en Prueba de Esfuerzo (A), y con el puntaje Duke Activity Status Index (DASI) (B). Los factores de riesgo considerados fueron hipertensión arterial, dislipidemia, diabetes, tabaquismo y obesidad. La comparación se hizo mediante prueba no-paramétrica de Mann-Whitney.

sujetos fueron referidos a protocolo Bruce $(93.7 \%)$. La mediana del DASI fue de 52,9 (IQR: 44,7-58,2), significativamente más alto en varones. La mediana (IQ) de METs por DASI fue de 9,2 (8,2-9,8) en la población total, versus la mediana de METs estimados en la PE de 10,2 (8,6-13,3). El VO2máx estimado por PE fue 30.4 $(24,8-34,7)$, y por DASI fue $32,3(28,8-34,6)$ (Tabla 2$)$.
Un 95.8\% de los cuestionarios fueron completados correctamente. El promedio de tiempo tomado para su llenado fue 2,5 minutos. La consistencia interna del cuestionario medida a través de la prueba alfa de Cronbach fue 0.72 , valor considerado aceptable para confiabilidad general de un cuestionario. Igual valor se obtuvo al aplicar la Formula 20 de Kuder Richardson (0.72).

\begin{tabular}{|c|c|c|c|c|}
\hline \multirow[t]{2}{*}{ Variable } & Total & Mujeres & Hombres & $P *$ \\
\hline & $\mathrm{N}=480$ & $\mathrm{~N}=181$ & $N=299$ & \\
\hline Edad (años) & $52(43-62)$ & $52(44-62)$ & $51(43-62)$ & NS * \\
\hline Hipertensión (\%) & $211(44.0)$ & $81(44.8)$ & $130(43.5)$ & NSt \\
\hline Diabetes (\%) & $102(21.2)$ & 39 (21.5) & $63(21.1)$ & NSt \\
\hline Dislipidemia (\%) & $165(34.4)$ & $53(29.3)$ & $112(37.5)$ & $0.07 \dagger$ \\
\hline Tabaquismo (\%) & $64(13.3)$ & $26(14.4)$ & 38 (12.7) & NS† \\
\hline Obesidad (\%) & $110(22.9)$ & $47(26.0)$ & $63(21.1)$ & NSt \\
\hline IMC (kg/m2) & $27.3(24.9-29.7)$ & $26.8(24.6-30.5)$ & $27.5(25.3-29.5)$ & NS * \\
\hline Enfermedad Coronaria (\%) & $74(15.4)$ & $15(8.3)$ & 59 (19.7) & $<0.001 \dagger$ \\
\hline Puntaje DASI & $52,9(44,7-58,2)$ & $50,7(37,2-58,2)$ & $58,2(50,7-58,2)$ & $<0.0001^{*}$ \\
\hline VO2máx DASI (ml/kg per min) & $32,4(28,8-34,6)$ & $31,4(25,6-34,6)$ & $34,6(31,4-34,6)$ & $<0.0001^{*}$ \\
\hline METs DASI & $9,2(8,2-9,8)$ & $8,9(7,3-9,8)$ & $9,8(8,9-9,8)$ & $<0.0001^{*}$ \\
\hline VO2máx PE (ml/kg per min) & $30,4(24,8-34,7)$ & $26,3(21,2-31,3)$ & $31,8(28,1-37,0)$ & $<0.0001^{*}$ \\
\hline METs PE & $10,2(8,6-13,3)$ & $10(7,1-10,2)$ & $11,7(10-13,5)$ & $<0.0001^{*}$ \\
\hline
\end{tabular}

Resultados presentados como frecuencia (porcentaje), y como mediana ( $p 50$ ) con rangos intercuartiles ( $p 25-p 75$ ) o como promedio con desviación estándar según distribución de valores. * Mann-Whitney signed-rank test † Fisher exact test DASI: Duke activity status index / VO2max: consumo de oxígeno máximo / METs: equivalentes metabólicos. 
A

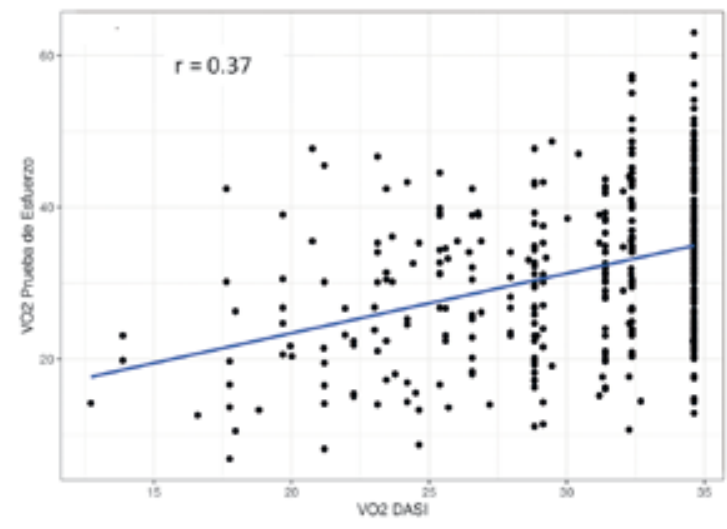

B

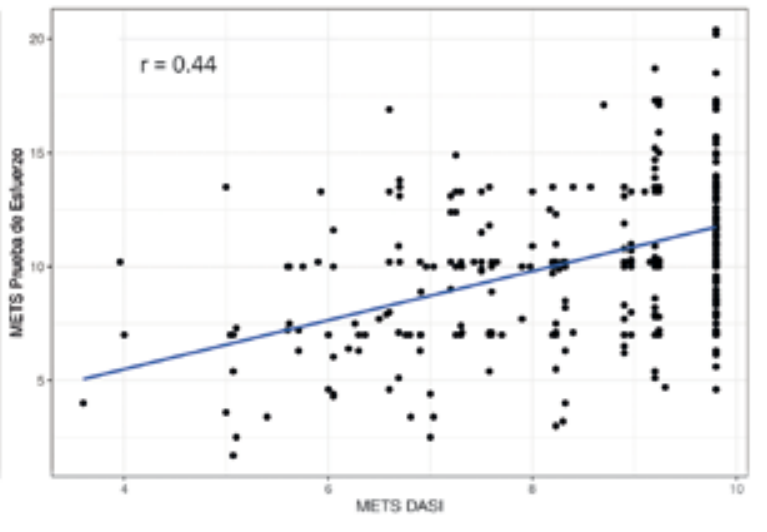

Correlación entre la estimación de Capacidad Funcional por Cuestionario DASI y Prueba de Esfuerzo Máxima. A- Correlación del Consumo de Oxígeno , B-Correlación con Equivalentes Metabólicos

DASI: Duke Activity Status Index; METs: equivalentes metabólicos; VO2 max: consumo de óxigeno máximo

En el análisis de carga de FRCV, se observó una asociación significativa $(\mathrm{p}<0.0001)$ entre el número de FRCV con METs alcanzados en PE, y con puntaje DASI ( $\mathrm{p}<$ 0.0001) (Figura 1). En la Figura 2 se muestra el análisis de regresión lineal realizado entre el VO2máx predicho por DASI y el alcanzado en PE, observándose una correlación de $\mathrm{r}=0.37$ ( $\mathrm{p}<0.001)$. A su vez, la correlación para los METS estimados por DASI y alcanzados en PE máxima fue $\mathrm{r}=0,44(\mathrm{p}<0.001)$. Adicionalmente, se realizó un modelo de regresión lineal simple ocupando METs en escala logarítmica. La ecuación basada en los datos chilenos quedó de la forma METs $=\mathrm{e} 0.015057 \times$ DASI + 1.564858. En la Figura 3 se observan los gráficos con función de suavizamiento LOESS.

En la Tabla 3 se expresan los valores obtenidos a través del modelo de regresión lineal Bayesiano, que entregan una distribución predictiva de METs para valores del DASI. De este modo, una persona con un puntaje DASI de 40 debería alcanzar 8.7 METs en una PE, con intervalo de credibilidad "highest probability density" (HPD) de 4.8 a 15.7. Finalmente, la Figura 4 ilustra la distribución predictiva de alcanzar más de 3, 6 y 9 METs en un PE

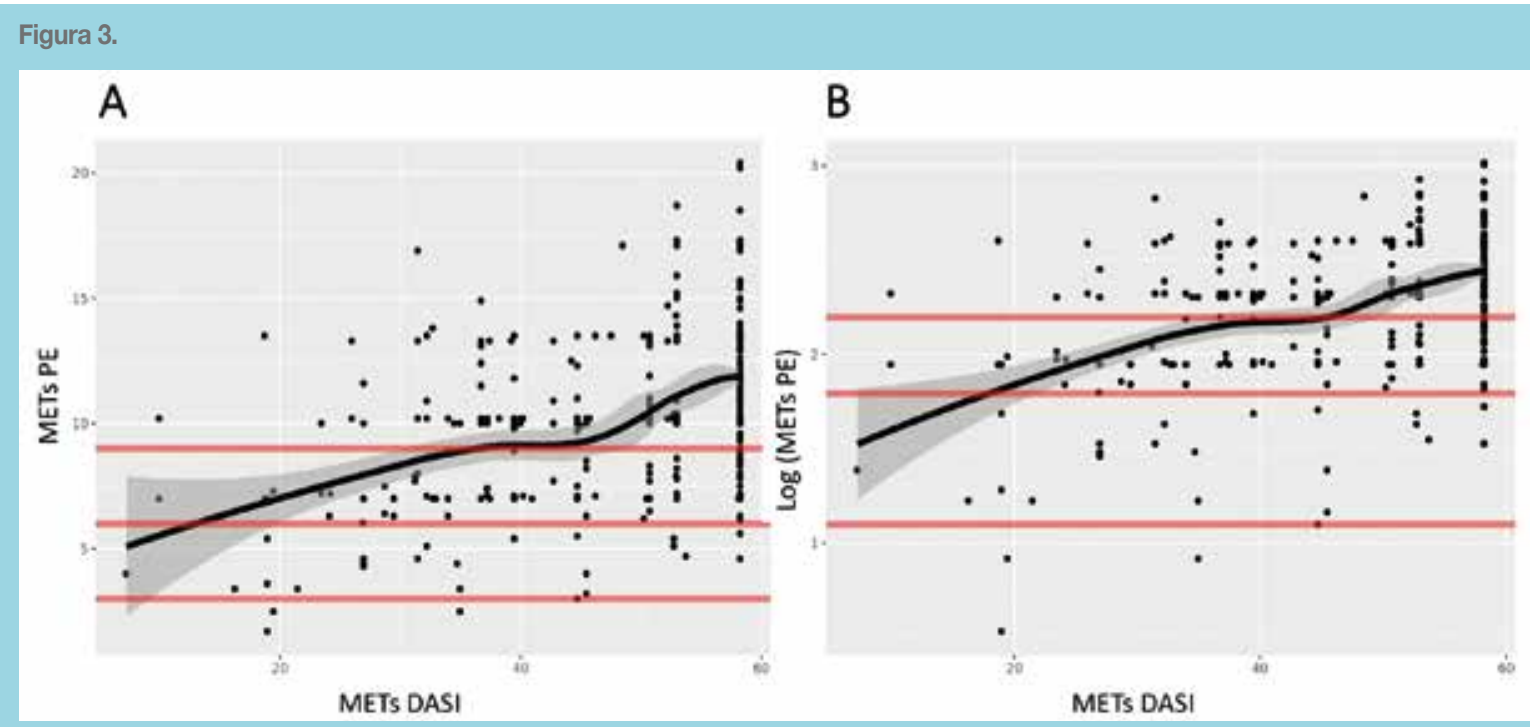

Suplementaria. Gráficas de Correlación en Escala Lineal (A) y Logarítmica (B) entre Capacidad Funcional en Prueba de Esfuerzo y puntuación DASI . Las líneas horizontales rojas representan puntos de corte de 3, 6, y 9 METs. 


\begin{tabular}{|c|c|c|c|c|c|c|c|c|c|c|c|c|}
\hline \multirow{2}{*}{$\begin{array}{l}\text { Puntaje } \\
\text { DASI }\end{array}$} & \multicolumn{3}{|c|}{$\begin{array}{c}\text { METS } \\
95 \% \text { IC HDP }\end{array}$} & \multicolumn{3}{|c|}{$\begin{array}{l}\text { Probabilidad } \\
>3 \text { METS } \\
95 \% \text { IC HDP }\end{array}$} & \multicolumn{3}{|c|}{$\begin{array}{c}\text { Probabilidad } \\
>6 \text { METS } \\
95 \% \text { IC HPD }\end{array}$} & \multicolumn{3}{|c|}{$\begin{array}{c}\text { Probabilidad } \\
>9 \text { METS } \\
95 \% \text { IC HDP }\end{array}$} \\
\hline & Media & Bajo & Alto & Media & Bajo & Alto & Media & Bajo & Alto & Media & Bajo & Alto \\
\hline 0 & 4.81 & 2.63 & 8.83 & 0.93 & 0.88 & 0.98 & 0.23 & 0.11 & 0.36 & 0.02 & 0.00 & 0.04 \\
\hline 5 & 5.17 & 2.86 & 9.79 & 0.96 & 0.93 & 0.99 & 0.31 & 0.18 & 0.44 & 0.04 & 0.01 & 0.07 \\
\hline 10 & 5.54 & 3.10 & 9.87 & 0.98 & 0.96 & 0.99 & 0.40 & 0.27 & 0.53 & 0.06 & 0.02 & 0.10 \\
\hline 15 & 5.98 & 3.41 & 11.41 & 0.99 & 0.98 & 1.00 & 0.50 & 0.37 & 0.61 & 0.09 & 0.04 & 0.14 \\
\hline 20 & 6.51 & 3.63 & 11.91 & 0.99 & 0.99 & 1.00 & 0.60 & 0.49 & 0.70 & 0.14 & 0.08 & 0.20 \\
\hline 25 & 6.98 & 4.02 & 13.31 & 1.00 & 0.99 & 1.00 & 0.69 & 0.61 & 0.77 & 0.20 & 0.14 & 0.27 \\
\hline 30 & 7.53 & 4.17 & 13.35 & 1.00 & 1.00 & 1.00 & 0.77 & 0.72 & 0.83 & 0.28 & 0.22 & 0.34 \\
\hline 35 & 8.05 & 4.36 & 14.26 & 1.00 & 1.00 & 1.00 & 0.84 & 0.80 & 0.88 & 0.36 & 0.31 & 0.42 \\
\hline 40 & 8.76 & 4.81 & 15.69 & 1.00 & 1.00 & 1.00 & 0.89 & 0.87 & 0.92 & 0.46 & 0.41 & 0.51 \\
\hline 45 & 9.36 & 5.11 & 16.64 & 1.00 & 1.00 & 1.00 & 0.93 & 0.91 & 0.95 & 0.56 & 0.52 & 0.60 \\
\hline 50 & 10.12 & 5.59 & 18.35 & 1.00 & 1.00 & 1.00 & 0.96 & 0.95 & 0.97 & 0.65 & 0.62 & 0.69 \\
\hline 55 & 10.89 & 5.93 & 19.57 & 1.00 & 1.00 & 1.00 & 0.98 & 0.97 & 0.99 & 0.74 & 0.71 & 0.78 \\
\hline 58 & 11.48 & 6.36 & 20.45 & 1.00 & 1.00 & 1.00 & 0.98 & 0.98 & 0.99 & 0.79 & 0.75 & 0.82 \\
\hline
\end{tabular}

en esta población. Así por ejemplo, un sujeto con puntaje DASI de 40, tiene una probabilidad de obtener un rendimiento superior a 3, 6, y 9 METs estimado en 1.0, 0.89 y 0.46 .

\section{Discusión:}

El presente estudio es el primer reporte en población chilena de la aplicación del cuestionario DASI como instrumento evaluador de CF. La traducción y adaptación al español fue bien recibida por los pacientes, con más de un $95 \%$ de ellos que respondieron la totalidad del cuestionario. El resultado del cuestionario DASI mostró una adecuada consistencia interna, y hubo una correlación positiva entre el VO2máx y los METS logrados en una PE limitada por síntomas con aquellos estimados por DASI.

El estudio demostró una correlación positiva de rango bajo-moderado, menor a la original de Hlatky, en el cual la determinación del VO2máx fue por test cardiopulmonar. En relación a estudios con PE, existen reportes como el de Coutinho-Myrrha y col., con una correlación mayor del DASI en población cardiópata en Brasil7. No obstante, nuestros resultados son concordantes con otros estudios con PE. Es así como en Norteamérica, Coute y col., observaron en análisis de regresión lineal un $\mathrm{r}^{2}$ de 0,38 del DASI con METs estimados por PE máxima, en población de ambos sexos ${ }^{17}$. A la vez, Bairey-Merz y col., en un estudio con mujeres, obtuvo una correlación positiva de METs estimados por DASI con aquellos alcanzados en una PE máxima (r: $0.31, p<0.002)^{18}$. Esa correlación fue catalogada como apropiada y superior a la obtenida cuando aplicaban el cuestionario PEPI-Q (Postmenopausal Estrogen and Progesterone Intervention physical activity Questionnaire), por lo que los autores recomiendan la utilización del DASI en mujeres para estimar $\mathrm{CF}^{18}$. A la luz de esta información, es comprensible que el DASI se haya incorporado en el algoritmo de la AHA para estudio de cardiopatía isquémica en la mujer 6 .

La utilidad principal del DASI radica en ser un instrumento de estimación de la CF. La CF se asocia a mortalidad CV. Así, en un estudio con determinación de VO2máx a través de test cardiopulmonar, se objetivó que cada incremento en 1 MET se asocia a una reducción de un $16 \%$ en mortalidad $\mathrm{CV}^{19}$. Otros estudios han demostrado que incorporar la determinación de CF con METs logrados en PE mejora la predicción de riesgo CV en sujetos asintomáticos, adicionalmente al uso de ecuaciones de riesgo ${ }^{20,21}$. La manera más objetiva de estimar $\mathrm{CF}$ es 


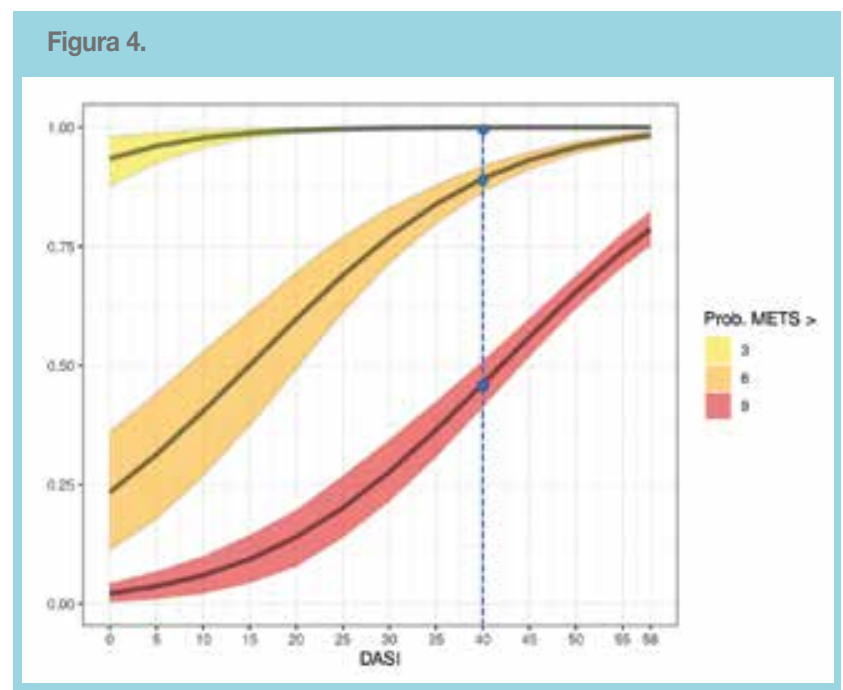

Figura 4. Gráfica de Distribución Predictiva de la Probabilidad de obtener un Rendimiento Superior a 3, 6, 9 METs en función de una puntuación DASI. Las bandas de colores representan intervalos de credibilidad HPD (Highest probability Density) de 95\%. La línea punteada azul demarca una puntuación DASI de 40. Los círculos demarcan la probabilidad de obtener unrendimiento superior a 3, 6 y 9 METs con DASI 40 .

a través de la medición directa del VO2máx mediante test cardiopulmonar. Sin embargo, esta técnica es sofisticada y de difícil acceso, por lo que se destina a portadores de insuficiencia cardíaca o deportistas de alto rendimiento 22,23 . Por otra parte, la PE es una alternativa accesible, que determina en forma indirecta el VO2máx., a través de ecuaciones que consideran el tiempo alcanzado. La prueba debe continuarse hasta la máxima carga tolerada, independiente de llegar al $85 \%$ de la frecuencia cardíaca máxima teórica, para lo cual es importante que el evaluador seleccione bien el protocolo de ejercicio. Por lo anterior, el contar con un cuestionario como DASI, y considerar la tabla de predicción de METs aportada, puede ser de utilidad en el momento de indicar o no una PE. Al respecto, el consenso del AHA sobre sospecha de enfermedad coronaria de la mujer menciona que, ante la incapacidad de alcanzar 5 METS en actividades de la vida diaria por DASI, es recomendable seleccionar un test de estrés farmacológico por sobre una PE convencional $^{6}$. Creemos que nuestra traducción del cuestionario evaluado puede entregar una herramienta práctica para dicha valoración.

El DASI le entrega al clínico una orientación indirecta de la CF del paciente, es decir no reemplaza a la PE, y se recomienda utilizarlo como complemento de ésta. No obstante, hay que considerar que algunos pacientes no pueden realizar una PE, y el DASI puede ser de mucha utilidad en estos casos. Es así, como el DASI ha sido incorporado por anestesiólogos como parte de la evaluación preanestésica en el ítem de $\mathrm{CF}$, llegando a ser recomendado dentro de la evaluación preoperatoria de riesgo CV para cirugía no cardíaca ${ }^{24,25}$. Recordemos que dentro de la evaluación preoperatoria se determina si el paciente tiene una CF de al menos $4 \mathrm{METs}^{26}$. De este modo, sino se dispone de una PE, es de utilidad acceder a un instrumento práctico como el DASI, que orienta sobre la CF estimada en METs.

Igualmente, el DASI es de utilidad en población con insuficiencia cardíaca. Al respecto, Fan y col. validaron las propiedades psicométricas del DASI como estimador de la $\mathrm{CF}$ en estos pacientes, valorándolo por ser una herramienta fácil de aplicar ${ }^{27}$. Además, en portadores de insuficiencia cardíaca se ha observado como el puntaje DASI difiere significativamente entre pacientes con eventos $\mathrm{CV}$ versus aquellos sin eventos CV. ${ }^{28}$ En pacientes con enfermedad coronaria estable también se ha reportado que proporciona un valor pronóstico independiente para eventos CV mayores a largo plazo, comparable al valor pronóstico de biomarcadores cardíacos ${ }^{19}$. Así, Shaw y col. indican que los METs por DASI serían un estimador independiente de riesgo de infarto miocárdico y/o muerte, incluso ajustado por edad, IMC, FRCV, historia de enfermedad coronaria y extensión de ésta en la angiografía ${ }^{20}$. De este modo, el aplicar el DASI en nuestro práctica clínica puede ser útil, no sólo como un elemento de estimación de $\mathrm{CF}$, sino también para predicción de riesgo $\mathrm{CV}$ en poblaciones como las mencionadas.

Nuestro estudio tiene limitaciones. No se realizó determinación directa de VO2máx por test cardiopulmonar, que sería el estándar de oro. No obstante, la mayoría de los estudios de validación del DASI han utilizado PE. Adicionalmente, las respuestas al cuestionario pueden variar por nivel educacional, factor que no analizamos en el estudio. Otra limitación fue una mediana de DASI elevado, y un porcentaje bajo de sujetos con $<6$ METs. Esto puede deberse al hecho de haber incorporado una amplia población, no focalizada a pacientes con insuficiencia cardíaca.

En conclusión, en este estudio se demuestra que el cuestionario DASI en español y adaptado a población chilena presenta una correlación positiva con VO2máx y METs estimados en PE. Estos resultados son de utilidad para la práctica clínica, puesto que permiten estimar la CF de un paciente por esta simple y rápida vía; Esta estimación es trascendente considerando que la $\mathrm{CF}$ es un valioso determinante para decisiones clínicas, y además, es un predictor de riesgo CV a largo plazo. 


\section{Referencias:}

1) ARENA R, MYERS J, WILLIAMS MA, GULATI M, KLIGFIELD P, BALADY GJ, et al; American Heart Association Committee on Exercise, Rehabilitation, and Prevention of the Council on Clinical Cardiology; American Heart Association Council on Cardiovascular Nursing. Assessment of functional capacity in clinical and research settings: a scientific statement from the American Heart Association Committee on Exercise, Rehabilitation, and Prevention of the Council on Clinical Cardiology and the Council on Cardiovascular Nursing. Circulation. 2007 Jul 17;116(3):329-43.

2) LAUKKANEN JA, RAURAMAA R, SALONEN JT, KURL $\mathrm{S}$. The predictive value of cardiorespiratory fitness combined with coronary risk evaluation and the risk of cardiovascular and all-cause death. J Intern Med. 2007 Aug;262(2):263-72

3) ROSS R, BLAIR S, ARENA R, CHURCH T, DESPRÉS JP, FRANKLIN B. et al. Importance of Assessing Cardiorespiratory Fitness in Clinical Practice: A Case for Fitness as a Clinical Vital Sign. A Scientific Statement from the American Heart Association. Circulation 2016; 134:e653-e699. DOI:10.1161/CIR.0000000000000461

4) JETTÉ M, SYDNEY K, BLÜMCHEN G. Metabolic equivalents (METS) in exercise testing, exercise prescription and evaluation of functional capacity. Clin Cardiol 1990; 13:555-565

5) HLATKY M, BOINEAU R, HIGGINBOTHAM M, LEE KERRY, DANIEL M , CALIFF R, et al. A brief self administered questionnaire to determine functional capacity ( The Duke Activity Status Index) Am J. Cardiol 1989;64: 651-654

6) MIERES J, GULATI M, BAIREY MERZ N, BERMAN D, GERBER T, HAYES $S$ et al. Role of Noninvasive Testing in the Clinical Evaluation of Women With Suspected Ischemic Heart Disease: A Consensus Statement From the American Heart Association. Circulation 2014;130: 350-379

7) COUTINHO-MYRRHA M, DIAZ R, FERNANDES A, ARAÚJO C, HLATKY M, PEREIRA D et al. Duke Activity Status Index for Cardiovascular Diseases: Validation of the Portuguese Translation. Arq. Bras. Cardiol, 2014; 102(4): 383-390
8) SANCHEZ EM, VERA CY, NAVAS CM, ORTIZ SD, RODRIGUEZ C, VARGAS DM, y col.Validación para Colombia del cuestionario para la "Medición de capacidad funcional en pacientes con falla cardíaca". Rev Colomb Cardiol 2018;25: 356-365

9) FLETCHER GF, ADES PS, KLIGFIELD P, ARENA R, BALADY G, BITTNER V et al on behalf of the American Heart Association Exercise, Cardiac Rehabilitation, and Prevention Committee of the Council on Clinical Cardiology, Council on Nutrition, Physical Activity and Metabolism, Council on Cardiovascular and Stroke Nursing, and Council on Epidemiology and Prevention. Exercise Standards for Testing and Training: a scientific statement from the American Heart Association. Circulation. 2013;128:873-934,

10) ARONSON D, SHEIKH-AHMAD M, AVIZOHAR O, KERNER A, SELLA R, BARTHA P et al. C- Reactive protein is inversely related to physical fitness in middle -aged subjects. Atherosclerosis 2004;176:173-179

11) FOSTER C, POLLOCK ML, ROD JL, DYMOND DS, WIBLE G, SCHMIDT D. Evaluation of Functional Capacity during Exercise Radionuclide Angiography. Cardiology 1983;70:85-93

12) GIBSON AL, WAGNER DR, HEYWARD VH. Advanced Fitness Assessment and Exercise Prescription, Eighth edition. Champaign, Illinois: Human Kinetics, 2019 . ISBN:978-14925-6134-7

13) FOSTER C, JACKSON AS, POLLOCK ML, TAYLOR MM, HARE J, SENNETT SM et al. Generalized equations for predicting functional capacity from treadmill performance. Am Heart J 1984; 107:1229-1234.

14) WILMORE, JACK H. \& COSTILL, DAVID. L. 2004. Physiology of Sport and Exercise, 3 rd ed. Human Kinetics, Champaign,III; Leeds

15) NORTON K., NORTON L, SADGROVE D. Position statement on physical activity and exercise intensity. J Sci Med 
Sport 2010;13:496-502. doi: 10.1016/j.jsams.2009.09.008

16) HASKELL WL, LEE IM, PATE RR, POWELL KE, BLAIR SN, FRANKLIN BA et al. Physical Activity and Public Health. Updated Recommendation for Adults from the American College of Sports Medicine and the American Heart Association. Circulation 2007;116:1081-1093

17) COUTE RA, EHRENFELD JM, GUPTA DK, TEREKHOV MA, WANDERER JP. Electronically self-assessed functional capacity and exercise testing: A comparison of the Duke Activity Status Index and Patient-Reported Outcomes Measurement Information System tools. Am Heart J. 2017; 188:82-86.

18) BAIREY MERZ CN, OLSON M, MCGORRAY S, PAKSTIS DL, ZELL K, RICKENS CR et al. Physical activity and functional capacity measurement in women: a report from the NHLBI-sponsored WISE study. J Womens Health Gend Based Med. 2000;9(7):769-777. doi:10.1089/15246090050147745

19) IMBODEN MT, HARBER MP, WHALEY MH, FINCH WH, BISHOP DL, KAMISKY LA. Cardiorespiratory fitness and mortality in healthy men and women . J Am Colll Cardiol 2018; 72: 2283-2292.

20) ISRAEL A, KIVITY S, SIDI Y, SEGEV S, BERKIVITCH A, KLEMPFNER $\mathrm{R}$ et al. Use of exercise capacity to improve SCORE risk prediction model in asymptomatic adults. Eur Heart J 2016:37: 2300-2306.doi:10.1093/eurheartj/ehw053

21) ACEVEDO M, VALENTINO G, BUSTAMENTE MJ, ORELLANA L, ADASME M, BARAONA F et al. Cardiorespiratory fitness improves prediction of mortality of standard cardiovascular risk scores in a Latino population. Clinical Cardiology, 2020; 43 (10):1167-1174.

22) GUAZZI M, BANDERA F, OZEMEK C, SYSTROM D, ARENA R. Cardiopulmonary Exercise Testing. What is its Value? J Am Coll Cardiol 2017;70:1618-36

23) ZINNER C, SPERLICH B, WAHL P, MESTER J. Classification of selected cardiopulmonary variables of elite athletes of different age, gender, and disciplines during incremental exercise testing. Springer Plus 2015; 4: 544 doi: 10.1186/ s40064-015-1341-8

24) SEPÚLVEDA $\mathrm{P}$ ¿Qué entendemos por la Clasificación ASAPS? Gastroenterol.latinoam 2013;24:38-43

25) WIJEYSUNDERA DN, BEATTIE WS, HILLIS GS, ABBOTT TEF, SHULMAN MA, ACKLAND GL et al. Integration of the Duke Activity Status Index into preoperative risk evaluation: a multicenter prospective cohort study. Br J Anaesth. 2020 Mar;124(3):261-270. doi: 10.1016/j.bja.2019.11.025

26) KRISTENSEN SD, KNUUTI J, SARASTE A, ANKER S, BØTKER HE, HERT SD, et al. 2014 ESC/ESA Guidelines on non-cardiac surgery: cardiovascular assessment and management: The Joint Task Force on non-cardiac surgery: cardiovascular assessment and management of the European Society of Cardiology (ESC) and the European Society of Anaesthesiology (ESA). Eur Heart J. 2014 ;35(35):2383-431.

27) FAN X, SUK LEE K, FRAZIER SK, LENNIE TA, MOSER DK. Psychometric testing of the Duke Activity Status Index in patients with heart failure. Eur J Cardiovasc Nurs, 2015, 214-221

28) PARISSIS JT, NIKOLAOU M, BIRMPA D, FARMAKIS D, PARASKEVAIDIS I, BISTOLA V, et al. Clinical and prognostic value of Duke's Activity Status Index along with plasma B-type natriuretic peptide levels in chronic heart failure secondary to ischemic or idiopathic dilated cardiomyopathy. Am J Cardiol. 2009;103(1):73-75.

29) TANG WH, TOPOL EJ, FAN Y, WU Y, CHO L, STEVENSON C et al Prognostic Value of Estimated Functional Capacity Incremental to Cardiac Biomarkers in Stable Cardiac Patients. J Am Heart Assoc 2014;3 (5):e000960/ doi: 10:10.116/ JAHA.114.00960.

30) SHAW LJ, OLSON MB, KIP K, KELSEY SF, JOHNSON BD, MARK DB, et al. The Value of Estimated Functional Capacity in Estimating Outcome. Results from the NHBLI Sponsored Women's Ischemia Syndrome Evaluation (WISE) Study. J Am Coll Cardiol 2006; 47:36S- 43S. 